\title{
Article \\ Effect of Mismatched Electron-Hole Effective Masses on Superfluidity in Double Layer Solid-State Systems
}

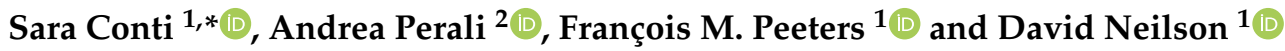 \\ 1 Department of Physics, University of Antwerp, Groenenborgerlaan 171, 2020 Antwerp, Belgium; \\ francois.peeters@uantwerpen.be (F.M.P.); david.neilson@uantwerpen.be (D.N.) \\ 2 Supernano Laboratory, School of Pharmacy, Università di Camerino, 62032 Camerino, Italy; \\ andrea.perali@unicam.it \\ * Correspondence: sara.conti@uantwerpen.be
}

Citation: Conti, S.; Perali, A.;

Peeters, F.M.; Neilson, D. Effect of Mismatched Electron-Hole Effective Masses on Superfluidity in Double Layer Solid-State Systems. Condens. Matter 2021, 6, 14. https://doi.org/ $10.3390 /$ condmat6020014

Academic Editor: Kenichi Kasamatsu

Received: 11 March 2021

Accepted: 2 April 2021

Published: 7 April 2021

Publisher's Note: MDPI stays neutral with regard to jurisdictional claims in published maps and institutional affiliations.

Copyright: (c) 2021 by the authors. Licensee MDPI, Basel, Switzerland. This article is an open access article distributed under the terms and conditions of the Creative Commons Attribution (CC BY) license (https:// creativecommons.org/licenses/by/ $4.0 /)$.

\begin{abstract}
Superfluidity has been predicted and now observed in a number of different electron-hole double-layer semiconductor heterostructures. In some of the heterostructures, such as GaAs and Ge-Si electron-hole double quantum wells, there is a strong mismatch between the electron and hole effective masses. We systematically investigate the sensitivity to unequal masses of the superfluid properties and the self-consistent screening of the electron-hole pairing interaction. We find that the superfluid properties are insensitive to mass imbalance in the low density BEC regime of stronglycoupled boson-like electron-hole pairs. At higher densities, in the BEC-BCS crossover regime of fermionic pairs, we find that mass imbalance between electrons and holes weakens the superfluidity and expands the density range for the BEC-BCS crossover regime. This permits screening to kill the superfluid at a lower density than for equal masses.
\end{abstract}

Keywords: superfluidity; electron-hole; exciton; BEC; BEC-BCS crossover; unequal masses; mass imbalance

\section{Introduction}

Pairing of fermions with significantly different masses is currently of great theoretical and experimental interest in condensed matter and nuclear physics. In such systems, there is potential to generate novel superfluid phenomena including a complex structure of the vortex state, with the vortex-core density depleted in a very different manner for the light and the heavy fermion components [1]. There is also the possibility of exotic superfluid phases across the BEC-BCS crossover. These include the Fulde-Ferrell-LarkinOvchinnikov [2] superfluid phase and the Sarma superfluid phase with two Fermi surfaces (breached pair phase) [3], and also possibly a Larkin-Ovchinnikov supersolid phase [4].

In ultracold atomic Fermi gases, Dy-K Fermi mixtures have been used to study massimbalanced strongly interacting Fermi-Fermi mixtures [5]. However, the predicted transition temperatures for exotic phases are inaccessibly low [6], even if evaporative cooling techniques are exploited.

Electron-hole double layer systems offer the intriguing possibility to generate massimbalanced fermionic systems in the solid state [7,8]. Transition temperatures for exotic phases are predicted to be a few Kelvin and thus experimentally accessible [9].

In these systems, the effects of the mass imbalance on superfluid properties can be explored throughout the BEC-BCS crossover, by tuning the density or the separation between the layers [10]. Thus mass imbalanced electron-hole double layer systems offer a rich playground to explore novel quantum effects and exotic superfluid phases.

We have previously included the effect of mass imbalance on the electron-hole superfluid phase diagram in GaAs [11] and Ge/Si [12] double quantum well systems. Other examples of candidate systems with mass imbalance are Transition Metal Dichalcogenide heterostructures [13] and InAs/GaSb double quantum wells [14]. In the present paper, we 
systematically investigate the sensitivity of the superfluid ground state properties to variations in the ratio of the electron and hole masses in a model double-layer solid-state system.

In the BCS weakly-coupled regime, a primary issue of unequal masses is the mismatched Fermi energies at equal densities. In the deep BEC strongly-coupled regime where the chemical potential becomes negative and the Fermi surfaces have collapsed, the electron-hole pairs are Boson-like, compact, and strongly bound, and the effect of unequal masses is much weaker.

Screening is known to play a central role in electron-hole superfluid properties, since the Coulombic pairing interaction is long-ranged [15]. There is an interplay between screening and the superfluid energy gap, since the superfluid gap blocks low-lying excitations out of the Fermi sea. This weakens the screening because those low-lying excitations are the excitations needed to screen the electron-hole interaction [16]. In the BEC regime, this small- $q$ suppression of screening leads to strong electron-hole pairing for $q \leq 2 k_{F}$ and, as a consequence, large superfluid gaps. In contrast, for the small superfluid gaps in what would be the BCS regime, superfluidity is completely suppressed by the screening. In the BEC-BCS crossover and BEC regimes, we will find that unequal masses mainly affect the screening of the electron-hole pairing interaction. Because of the suppression of superfluidity in the BCS regime, mismatch of the Fermi energies at equal densities is not a significant effect.

We systematically investigate these points for our model solid-state system as a function of the ratio of unequal electron and hole masses, in the two regimes of electronhole superfluidity, BEC and BEC-BCS crossover.

\section{Materials and Methods}

Without loss of generality, in our model system we will fix the electron effective mass $m_{e}=0.2 m_{0}$ and vary $m_{h}$, the hole effective mass. $m_{0}$ is the bare electron mass.

We calculate the zero temperature superfluid properties at equal electron and hole densities with a range of values for the effective mass ratio, $0<m_{h} / m_{e} \leq 1$.

\subsection{Mean Field Equations}

Considering only equal electron and hole densities $n_{e}=n_{h}=n$ at zero temperature, the superfluid energy gap $\Delta_{\mathbf{k}}$ can be determined from the mean-field equations of BCS theory, even in the strongly interacting BEC-BCS crossover and BEC regimes:

$$
\begin{gathered}
\Delta_{\mathbf{k}}=-\frac{1}{L^{2}} \sum_{\mathbf{k}^{\prime}} V_{\mathbf{k}-\mathbf{k}^{\prime}}^{s c} \frac{\Delta_{\mathbf{k}^{\prime}}}{2 E_{\mathbf{k}^{\prime}}}, \\
n=\frac{g_{s} g_{v}}{L^{2}} \sum_{\mathbf{k}} \frac{1}{2}\left(1-\frac{\xi_{\mathbf{k}}}{E_{\mathbf{k}}}\right), \\
E_{\mathbf{k}}=\sqrt{\xi_{\mathbf{k}}^{2}+\Delta_{\mathbf{k}}^{2}}, \quad \xi_{\mathbf{k}}=\frac{1}{2}\left(\xi_{\mathbf{k}}^{e}+\xi_{\mathbf{k}}^{h}\right), \quad \xi_{\mathbf{k}}^{\ell}=\frac{k^{2}}{2 m_{\ell}^{\star}}-\mu \quad \ell=e, h .
\end{gathered}
$$

$V_{\mathbf{k}-\mathbf{k}^{\prime}}^{s c}=V_{\mathbf{q}}^{s c}$ is the attractive screened electron-hole interaction which we specify later. $g_{s}=2$ and $g_{v}=1$ are the spin and valley degeneracy factors, and $\mu$ is the chemical potential taken equal for the electrons and holes.

For a given density $n$, Equations (1)-(3) are self-consistently solved for $\Delta_{\mathbf{k}}$ and $\mu$. We use the condensate fraction $C F$, defined as the ratio of the condensed pair density to the total density [17], to characterise the different regimes of the superfluid,

$$
C F=\frac{\sum_{\mathbf{k}} u_{\mathbf{k}}^{2} v_{\mathbf{k}}^{2}}{\sum_{\mathbf{k}} v_{\mathbf{k}}^{2}} .
$$

The Bogoliubov amplitudes are $u_{\mathbf{k}}^{2}=\frac{1}{2}\left(1+\frac{\xi_{\mathbf{k}}}{E_{\mathbf{k}}}\right)$ and $v_{\mathbf{k}}^{2}=\frac{1}{2}\left(1-\frac{\xi_{\mathbf{k}}}{E_{\mathbf{k}}}\right)$. 


\subsection{Self-Consistent Screening}

Because the electron-hole interaction is Coulombic and long-ranged, it is essential to include screening in $V_{\mathbf{q}}^{s c}$. To determine the screening in the presence of a superfluid, we evaluate the density response functions within the Random Phase Approximation [10]. Because of the different masses, there are distinct electron and hole normal polarizabilities [11]. The electron and hole normal polarizabilities in the presence of the superfluid are,

$$
\begin{aligned}
& \Pi_{e}(\mathbf{q})=-\frac{g_{s} g_{v}}{L^{2}} \sum_{\mathbf{k}}\left[\frac{u_{\mathbf{k}}^{2} v_{\mathbf{k}-\mathbf{q}}^{2}}{E_{\mathbf{k}-\mathbf{q}}^{+}+E_{\mathbf{k}}^{-}}+\frac{v_{\mathbf{k}}^{2} u_{\mathbf{k}-\mathbf{q}}^{2}}{E_{\mathbf{k}-\mathbf{q}}^{-}+E_{\mathbf{k}}^{+}}\right], \\
& \Pi_{h}(\mathbf{q})=-\frac{g_{s} g_{v}}{L^{2}} \sum_{\mathbf{k}}\left[\frac{u_{\mathbf{k}}^{2} v_{\mathbf{k}-\mathbf{q}}^{2}}{E_{\mathbf{k}-\mathbf{q}}^{-}+E_{\mathbf{k}}^{+}}+\frac{v_{\mathbf{k}}^{2} u_{\mathbf{k}-\mathbf{q}}^{2}}{E_{\mathbf{k}-\mathbf{q}}^{+}+E_{\mathbf{k}}^{-}}\right] .
\end{aligned}
$$

The anomalous polarizability for the density response of the superfluid electron-hole pairs is,

$$
\Pi_{a}(\mathbf{q})=\frac{g_{s} g_{v}}{L^{2}} \sum_{\mathbf{k}} \frac{\Delta_{\mathbf{k}}}{2 E_{\mathbf{k}}} \frac{\Delta_{\mathbf{k}-\mathbf{q}}}{2 E_{\mathbf{k}-\mathbf{q}}}\left[\frac{1}{E_{\mathbf{k}-\mathbf{q}}^{-}+E_{\mathbf{k}}^{+}}+\frac{1}{E_{\mathbf{k}-\mathbf{q}}^{+}+E_{\mathbf{k}}^{-}}\right]
$$

where

$$
E_{\mathbf{k}}^{ \pm}=E_{\mathbf{k}} \pm \delta \xi_{\mathbf{k}}, \quad \delta \xi_{\mathbf{k}}=\frac{1}{2}\left(\xi_{\mathbf{k}}^{h}-\xi_{\mathbf{k}}^{e}\right)
$$

In the long-wavelength limit $(q=0)$ we obtain from Equations (5)-(7):

$$
\Pi_{e}(q=0)=\Pi_{h}(q=0)=-\Pi_{a}(q=0)=-\frac{g_{s} g_{v}}{L^{2}} \sum_{\mathbf{k}} \frac{\Delta_{\mathbf{k}}^{2}}{2 E_{\mathbf{k}}^{2}}\left[\frac{1}{2 E_{\mathbf{k}}}+\frac{1}{2 E_{\mathbf{k}}}\right] .
$$

Note that the only mass parameter entering in Equation (9) is the reduced mass $m_{r}$.

The expression for the static screened electron-hole interaction for unequal masses in the presence of a superfluid is,

$$
V_{\mathbf{q}}^{s c}=\frac{V_{\mathbf{q}}^{D}}{1-\left[\Pi_{e}(\mathbf{q})+\Pi_{h}(\mathbf{q})\right] V_{\mathbf{q}}^{S}+2 V_{\mathbf{q}}^{D} \Pi_{a}(\mathbf{q})+\left[\left(V_{\mathbf{q}}^{s}\right)^{2}-\left(V_{\mathbf{q}}^{D}\right)^{2}\right]\left[\Pi_{e}(\mathbf{q}) \Pi_{h}(\mathbf{q})-\Pi_{a}^{2}(\mathbf{q})\right]} .
$$

$V_{\mathbf{q}}^{S}$ is the bare electron (hole) Coulomb repulsion within one layer, and $V_{\mathbf{q}}^{D}$ is the bare attraction between the electrons and holes in opposite layers separated by a distance $d$ :

$$
V_{\mathbf{q}}^{S}=\frac{2 \pi e^{2}}{\epsilon} \frac{1}{|\mathbf{q}|} ; \quad V_{\mathbf{q}}^{D}=-\frac{2 \pi e^{2}}{\epsilon} \frac{e^{-d|\mathbf{q}|}}{|\mathbf{q}|} .
$$

For our model system we will take $d=3 \mathrm{~nm}$, and dielectric constant for a $\mathrm{Si} / \mathrm{Ge}$ bilayer system [12], $\epsilon=13.7 \epsilon_{0}$, where $\epsilon_{0}$ is the vacuum dielectric constant.

The screened interaction in the presence of the superfluid, Equation (10), is determined self-consistently by calculating the polarizabilities Equations (5)-(7) using the $\Delta_{\mathbf{k}}$ and $\mu$ determined in the preceding iteration from Equations (1)-(3).

\subsection{Transition Temperature}

The superfluid transition temperature in this quasi-2D system is given by the BerezinskiiKosterlitz-Thouless (BKT) transition temperature [18],

$$
T_{B K T}=\frac{\pi}{2} \frac{\hbar^{2}}{M} \frac{n}{g_{s} g_{v}} .
$$

The transition temperature is linearly proportional to the carrier density $n$, and inversely proportional to the mass of the pairs $M=m_{e}+m_{h}$. 


\section{Results}

\subsection{Superfluid Properties}

The superfluid condensate fraction $C F$ (Equation (4)) is used to characterise the different superfluid regimes [19]. In the BCS regime, $C F \leq 0.2$, in the BEC-BCS crossover regime $0.2<C F<0.8$, and in the $\mathrm{BEC}$ regime, $C F \geq 0.8$.

Figure 1a shows the evolution of the condensate fraction with density $n$ for different values of $m_{h} / m_{e}$. At small densities, all the systems are in the BEC regime. As the density increases, they move out of the BEC regime into the BEC-BCS crossover regime. However, before the BCS regime can be reached, the screening becomes so strong and weakens the electron-hole pairing interaction so much that superfluidity is suppressed. Thus the superfluid exists only below some onset density $n_{0}$ [20].
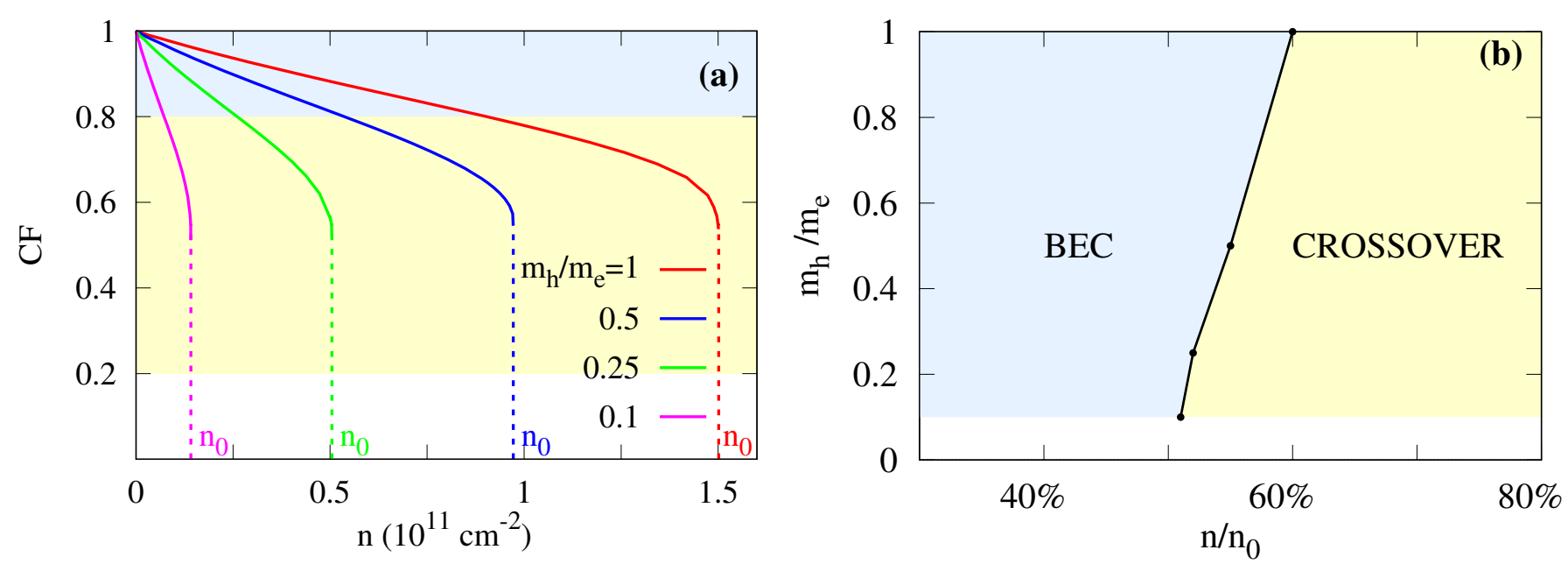

Figure 1. (a) Condensate fraction $C F$ as a function of density $n$ for different values of the mass ratio $m_{h} / m_{e}$. The blue shaded area is the BEC regime, the yellow area the BEC-BCS crossover regime and the unshaded area is what would be the BCS regime. The respective onset densities $n_{0}$ are indicated. (b) Relative percentage of the superfluid density range occupied by the BEC regime as function of $m_{h} / m_{e}$.

Comparing the behaviour of $C F$ for different values of $m_{h} / m_{e}$ in Figure $1 \mathrm{a}$, we see that for smaller $m_{h} / m_{e}$, the system leaves the BEC regime at a lower density and the onset density $n_{0}$ is also lower. Reducing $m_{h} / m_{e}$ while keeping $m_{e}$ fixed, decreases the reduced effective mass $m_{r}$, and this has the effect of increasing the effective Bohr radius $a_{B}^{\star}=\hbar^{2} 4 \pi \epsilon /\left(m_{r} e^{2}\right)$, decreasing the effective Rydberg energy $R y^{\star}=e^{2} /\left(4 \pi \epsilon 2 a_{B}^{\star}\right)$, and reducing the binding energy for an isolated electron-hole pair. Less strongly bound electronhole pairs weaken the strength of the superfluidity, narrow the density range for the BEC regime, and reduce $n_{0}$. It is interesting to note that the value $C F=0.5$ at the onset density remains the same, independent of $m_{h} / m_{e}$.

Figure $1 \mathrm{~b}$ shows the dependence on $m_{h} / m_{e}$ of the relative fraction of the superfluid density up to $n_{0}$ lying within the BEC regime. Large mass imbalances reduce the density range of the BEC regime, for which the bosonic character of the pairs dominates and the separate electron and hole masses are not important. Correspondingly, large mass imbalances expand the range of the BEC-BCS crossover regime, for which the fermionic nature of the pair components emerges and the superfluidity is sensitive to the separate electron and hole masses. The exotic superfluid phases which are associated with unequal masses, can therefore be expected to be restricted to the crossover regime. Thus, large mass imbalances should expand the phase space for these exotic phases. 
Figure 2 shows, the superfluid gap $\Delta$ as a function of equal electron and hole densities $n$ (Equation (1)). Above the onset density the superfluid gap is suppressed by strong screening. Screening is too strong to permit existence of the superfluid in the weak-coupled BCS regime, where by definition the superfluid gaps would be small compared with the Fermi energy [16]. As the density is lowered below $n_{0}$, the superfluid gap grows, passes through a maximum and then decreases to zero as $n$ decreases towards the zerodensity limit.

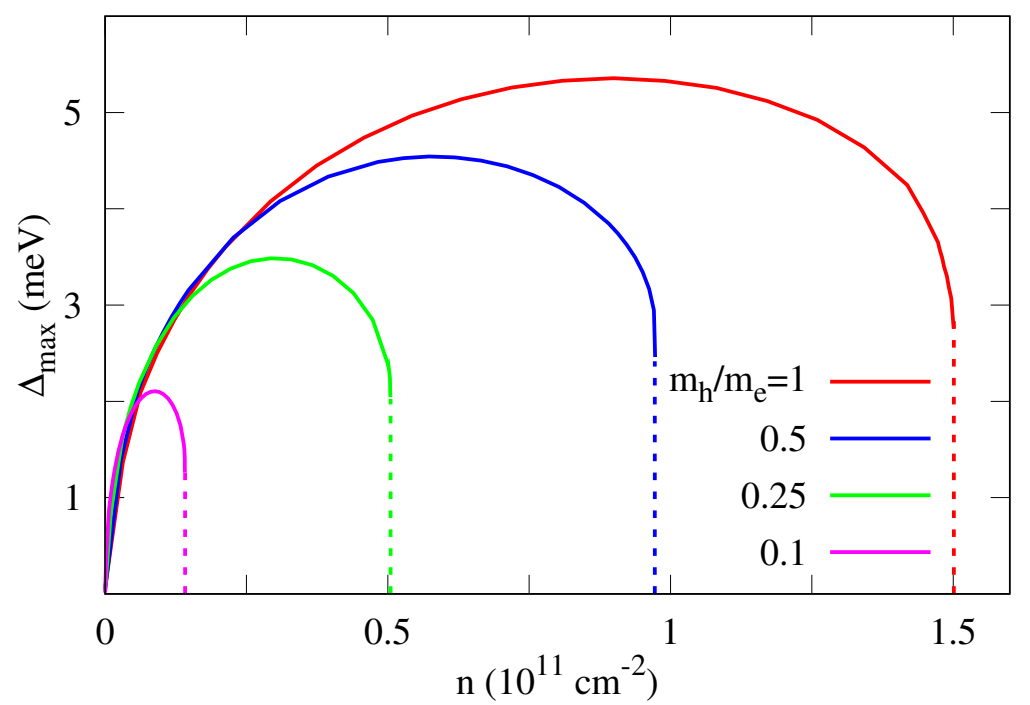

Figure 2. Maximum of the superfluid gap $\Delta_{\max }$ as function of density for different values of mass ratio $m_{h} / m_{e}$.

At very low densities the behaviour of $\Delta$ is insensitive to $m_{h} / m_{e}$, but at higher densities the maximum of $\Delta$ is lower as $m_{h} / m_{e}$ is made smaller, and the onset density $n_{0}$ also decreases with decreasing $m_{h} / m_{e}$. These properties of $\Delta$ reflect the behaviour we noted in the condensate fraction: the superfluidity weakens as $m_{h} / m_{e}$ is reduced below unity.

The transition temperature $T_{B K T}$ will be maximum at the onset density $n_{0}$ (see Equation (12)). Table 1 summarizes the effect of unequal masses on the onset density $n_{0}$ and the maximum transition temperature $T_{c}^{\max }$. Both $n_{0}$ and $T_{c}^{\max }$ are largest for equal masses.

Table 1. $m_{h} / m_{e}$ is the ratio between the masses. $n_{0}$ is the superfluid onset density. $T_{c}^{\max }$ is the maximum transition temperature.

\begin{tabular}{ccc}
\hline$m_{h} / m_{e}$ & $n_{\mathbf{0}}\left[\mathbf{1 0}^{\mathbf{1 1}} \mathbf{c m}^{-\mathbf{2}}\right]$ & $T_{c}^{\max }[\mathrm{K}]$ \\
\hline 1 & 1.5 & 2.6 \\
0.5 & 1.0 & 2.2 \\
0.25 & 0.5 & 1.4 \\
0.1 & 0.17 & 0.5 \\
\hline
\end{tabular}

\subsection{Polarizabilities}

To understand the sensitivity of $\Delta$ and $C F$ to $m_{h} / m_{e}$, we must examine the effect of unequal masses on the polarizabilities in the superfluid state. The polarizabilities are the building blocks of the screened electron-hole pairing interaction. As detailed in the Materials and Method section, the polarizabilities are sensitive to the size of the superfluid gap, so the screening must be determined self-consistently [10].

Figure 3 shows the polarizabilities for the superfluid state: the normal polarizabilities for electrons and holes, $\Pi_{e}(\mathbf{q})$ and $\Pi_{h}(\mathbf{q})$ (Equations (5) and (6)), and the anomalous polarizability for the superfluid electron-hole pairs, $\Pi_{a}(\mathbf{q})$ (Equation (7)). The panels show 
the polarizabilities for two densities, the first chosen in the BEC-BCS crossover regime near the onset density $n_{0}$ for each value of $m_{h} / m_{e}$, and the second in the deep BEC regime.

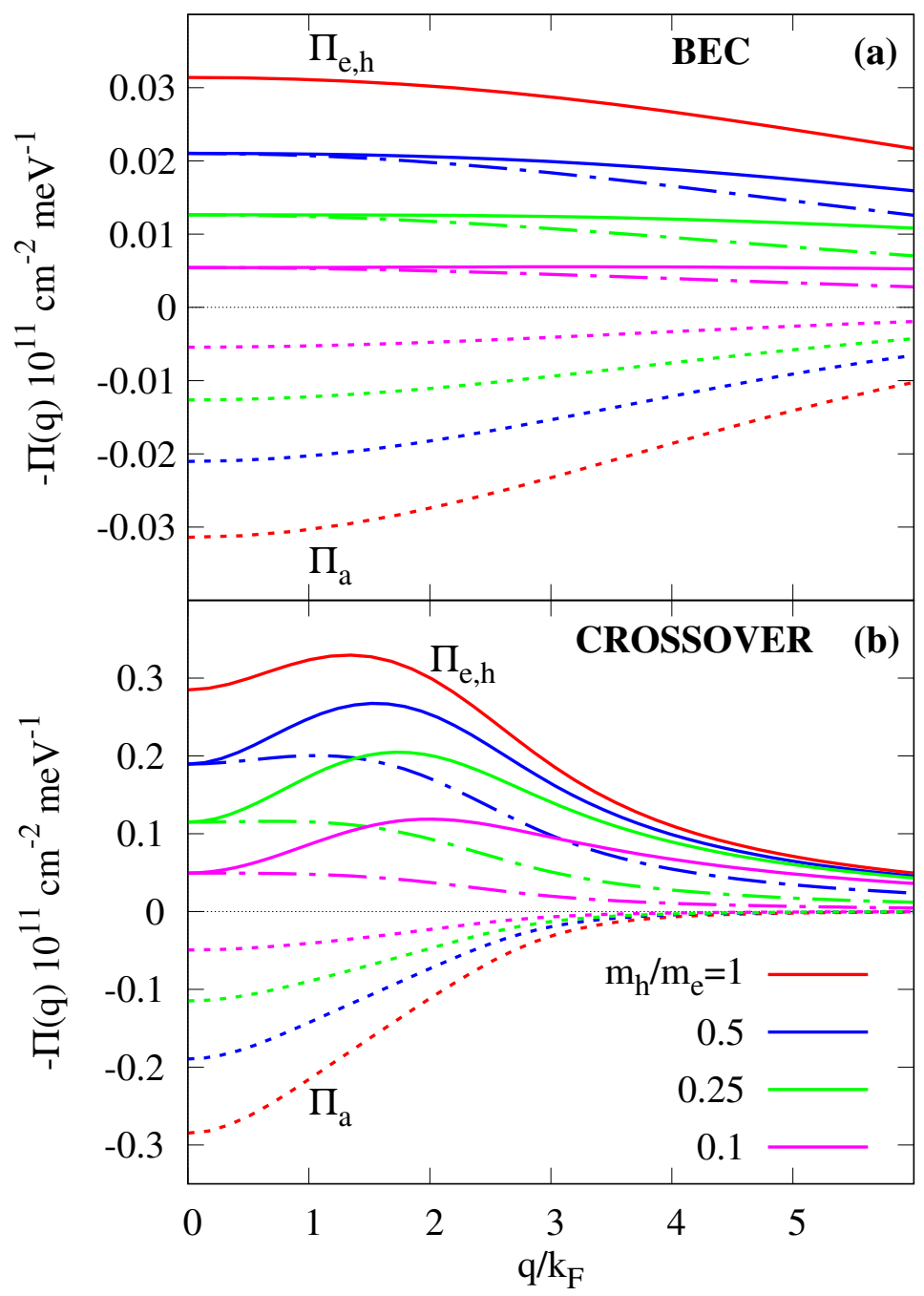

Figure 3. Polarizabilities in the superfluid state for different values of $m_{h} / m_{e}$. Normal polarizabilities $\Pi_{e}$ (solid line) and $\Pi_{h}$ (dashed-dotted line) for electrons and holes, respectively, and anomalous polarizability $\Pi_{a}(\mathbf{q})$ (dotted line). (a) For densities in the BEC regime with $C F=0.95$; (b)For densities in the crossover regime close to the onset density with $C F=0.5$.

We recall that for $q / k_{F} \leq 2$, the momentum transfer range affecting the screening, the polarizabilities $\Pi_{e}^{(N)}(\mathbf{q})$ and $\Pi_{h}^{(N)}(\mathbf{q})$ for the normal state are given by the respective density of states (Table 2). The normal polarizabilities $\Pi_{e}(\mathbf{q})$ and $\Pi_{h}(\mathbf{q})$ for the superfluid state shown in Figure 3 are seen to be much smaller. The physical argument is that a gap in the energy spectrum will exclude low-lying excited states from screening, and this very significantly weakens $\Pi_{\mathcal{e}}(\mathbf{q})$ and $\Pi_{h}(\mathbf{q})$.

Table 2. Polarizabilities in the normal state for different $m_{h} / m_{e}$ values. $\Pi_{e, h}^{(N)}(\mathbf{q})=m_{e, h} /\left(\pi \hbar^{2}\right)$ are the values for the electron and hole polarizabilities for $q / k_{F} \leq 2$. Units are $10^{11} \mathrm{~cm}^{-2} \mathrm{meV}^{-2}$.

\begin{tabular}{ccc}
\hline$m_{h} / m_{e}$ & $\Pi_{e}^{(N)}(\mathbf{q})$ & $\Pi_{h}^{(N)}(\mathbf{q})$ \\
\hline 1 & 0.8 & 0.8 \\
0.5 & 0.8 & 0.4 \\
0.25 & 0.8 & 0.2 \\
0.1 & 0.8 & 0.08 \\
\hline
\end{tabular}


In the screened interaction (Equation (10)), in addition to this reduction of the normal polarizabilities, there are significant cancellations of the $\Pi_{\mathcal{e}}(\mathbf{q})$ and $\Pi_{h}(\mathbf{q})$ with $\Pi_{a}(\mathbf{q})$, the anomalous polarizability. It is these effects together, that lead to a suppression of the screening and allow superfluidity [16].

In Figure $3, \Pi_{e}(q=0)=\Pi_{h}(q=0)=-\Pi_{a}(q=0)$ reflects the property that any non-zero gap in the low-lying excitation spectrum must eliminate screening in the longwavelength limit (see Equation (9)). The anomalous polarizability scales with the reduced mass of the electron-hole pairs. We are keeping $m_{e}$ fixed and decreasing $m_{h}$, so the system with equal masses has the largest reduced mass and the largest $\Pi_{a}(\mathbf{q})$.

Figure 3a shows in the deep BEC regime that superfluidity strongly weakens the normal polarizabilities $\Pi_{\mathcal{e}}(\mathbf{q})$ and $\Pi_{h}(\mathbf{q})$ in the momentum transfer range important for screening, $0<q / k_{F} \leq 2$. Appendix A demonstrates that the large superfluid gaps in the BEC regime overwhelm any effects arising from differences in the electron and hole masses. For this reason, the approximate equalities $\Pi_{\mathcal{e}}(\mathbf{q}) \simeq \Pi_{h}(\mathbf{q}) \simeq-\Pi_{a}(\mathbf{q})$ extend out beyond $q / k_{F} \simeq 2$, and the resulting cancellation eliminates screening in this regime. The physical reason is that the electron-hole pairs are strongly bound and are compact as compared with the average inter-particle spacing. This makes the pairs act as approximately neutral, thus minimising screening effects.

Figure $3 b$ shows at the onset density in the BEC-BCS crossover regime where the superfluid gap is relatively weak, that for unequal electron and hole masses $m_{e}>m_{h}$, $\Pi_{e}(\mathbf{q})$ is larger than $\Pi_{h}(\mathbf{q})$ for $q \neq 0$. Appendix B, Equations (A17) and (A18), show that for weak superfluid gaps, $\Pi_{e}(\mathbf{q})$ and $\Pi_{h}(\mathbf{q})$ are proportional to the electron and hole masses, respectively. In addition, Figure $3 b$ shows for small $q \neq 0$, that $\Pi_{h}(\mathbf{q}) \simeq-\Pi_{a}(\mathbf{q})$, but $\Pi_{e}(\mathbf{q})>-\Pi_{a}(\mathbf{q})$. From Equation (A21) in Appendix B, we see that $\Pi_{a}(\mathbf{q})$ is driven by the reduced mass $m_{r}$ and the lighter hole mass $m_{h}$, but it is not sensitive to the electron mass $m_{e}$. Because for large mass imbalance $m_{r} \simeq m_{h}, \Pi_{a}(\mathbf{q})$ will be closer to $\Pi_{h}(\mathbf{q})$ than to $\Pi_{e}(\mathbf{q})$. A net result is that in the crossover regime, $\Pi_{a}(\mathbf{q})$ will not be able to cancel $\Pi_{\mathcal{e}}(\mathbf{q})$, and so screening is not efficiently suppressed. The optimum configuration in this respect will be equal masses, since this maximises the cancellation of $\Pi_{a}(\mathbf{q})$ with both $\Pi_{e}(\mathbf{q})$ and $\Pi_{h}(\mathbf{q})$. This is the reason the onset density $n_{0}$ decreases as the mass difference increases (Figure 2).

\subsection{Excitation Energies}

The origin of the differences in functional behaviour between the electron and hole normal polarizabilities in the superfluid state can be better understood by examining the excitation spectrum of the systems. The behaviour of $\Pi_{e}(\mathbf{q})$ and $\Pi_{h}(\mathbf{q})$ is driven by changes the superfluid gap makes to the excitation spectrum when going from the normal to the superfluid state. We examine the same systems as in Figure 3.

Figure 4 shows for different $m_{h} / m_{e}$, the normal state spectrum $\xi_{\mathbf{k}}^{h}\left(\xi_{\mathbf{k}}^{e}\right)$ for the hole (electron) single-particle excitations and the corresponding superfluid state excitation spectrum $E_{\mathbf{k}}^{+}\left(E_{\mathbf{k}}^{-}\right)$(Equation (8)). We recall that the $E_{\mathbf{k}}^{ \pm}$differ by the mass difference factor $\pm \delta \xi_{\mathbf{k}}= \pm \frac{1}{2}\left(\xi_{\mathbf{k}}^{h}-\xi_{\mathbf{k}}^{e}\right)$. The colour-coded shaded areas show the low-energy states in $\xi_{\mathbf{k}}^{h}$ $\left(\xi_{\mathbf{k}}^{e}\right)$ that the superfluid gap excludes from $E_{\mathbf{k}}^{+}\left(E_{\mathbf{k}}^{-}\right)$. The excluded states are blocked from contributing to the polarizability, and this weakens the screening.

We recall in the BEC regime that $\Delta_{\mathbf{k}} / E_{F} \gg 1$ and $\Delta_{\mathbf{k}}$ is approximately constant from $k=0$ out to large $k / k_{F}$. In this way, $\Delta_{\mathbf{k}}$ blocks a very large number of low-lying excited states from the screening. Figure 4 a shows that the $E_{\mathbf{k}}^{ \pm}$is shifted up in energy by $\sim 20 E_{F}$ relative to $\xi_{\mathbf{k}}^{e, h}$. Since $\Delta_{\mathbf{k}} \gg \delta \xi_{\mathbf{k}}$, it follows that $E_{\mathbf{k}} \simeq E_{\mathbf{k}}^{-} \simeq E_{\mathbf{k}}^{+}$, so in the BEC regime the unequal masses have little effect on the polarizability properties.

In contrast, in the BEC-BCS crossover regime we find that excitation energies are sensitive to unequal masses. Figure $4 \mathrm{~b}$ is for a density in the crossover regime close to the onset density. The green panels show that as $m_{h} / m_{e}$ is decreased, $E_{\mathbf{k}}^{-}$moves down towards $\xi_{\mathbf{k}}^{e}$. This is because of the subtraction of $\delta \xi_{\mathbf{k}}$ that becomes larger with decreasing $m_{h} / m_{e}$ For this reason the maximum in $\Pi_{e}(\mathbf{q})$ becomes larger as the mass imbalance increases 
(Figure $3 b$ ). For the holes (blue panels) $\delta \xi_{\mathbf{k}}$ is added to $E_{\mathbf{k}}$, and moves $E_{\mathbf{k}}^{+}$up and away from $\xi_{\mathbf{k}}^{h}$ as $m_{h} / m_{e}$ decreases. As a net result, one can see that for electrons, the excluded states are reduced as $m_{h} / m_{e}$ decreases, while for holes the range of excluded states expands. Thus for unequal masses, the superfluid gap $\Delta_{\mathbf{k}}$ is less effective at blocking excitation states for electrons than for holes over the full range of $k / k_{F}$ important for screening.

BEC

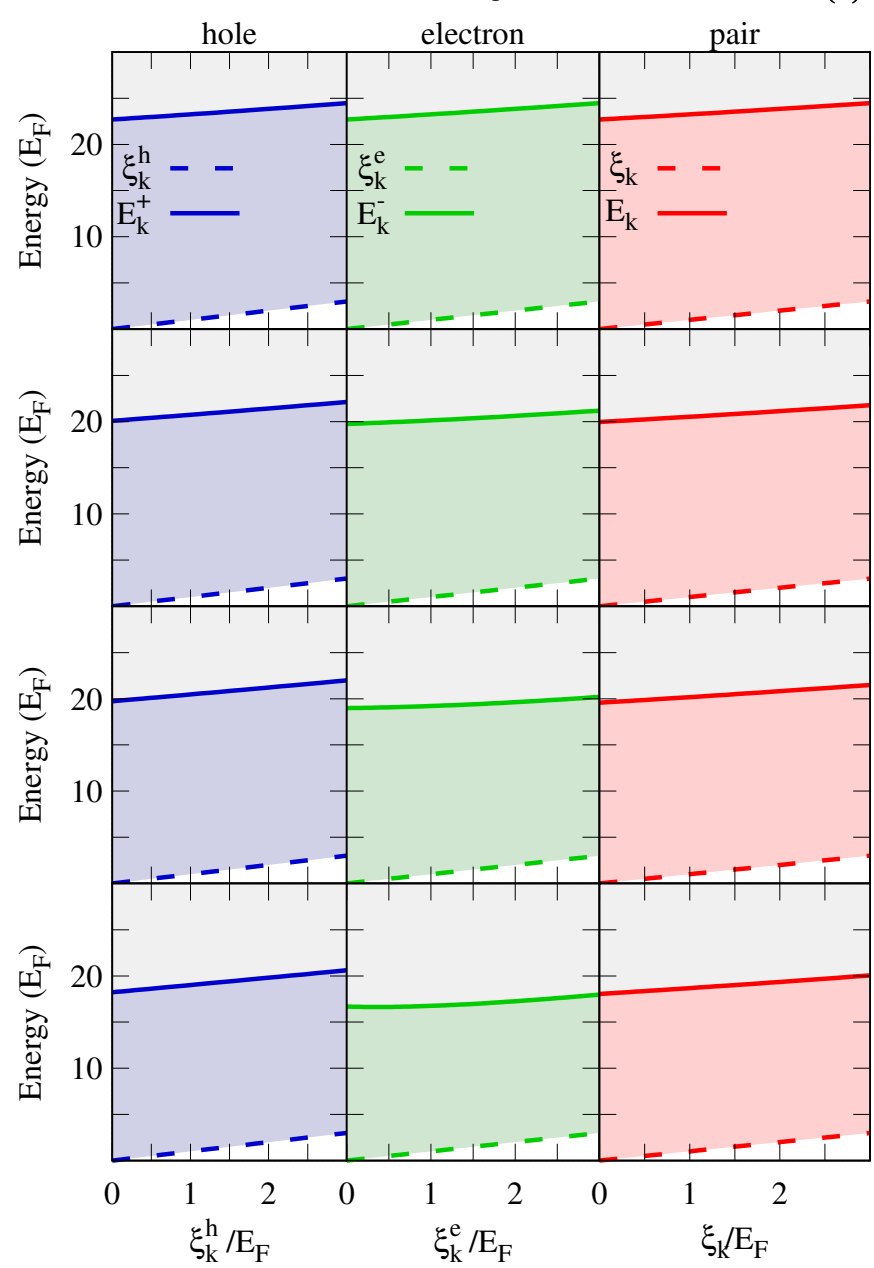

(a)

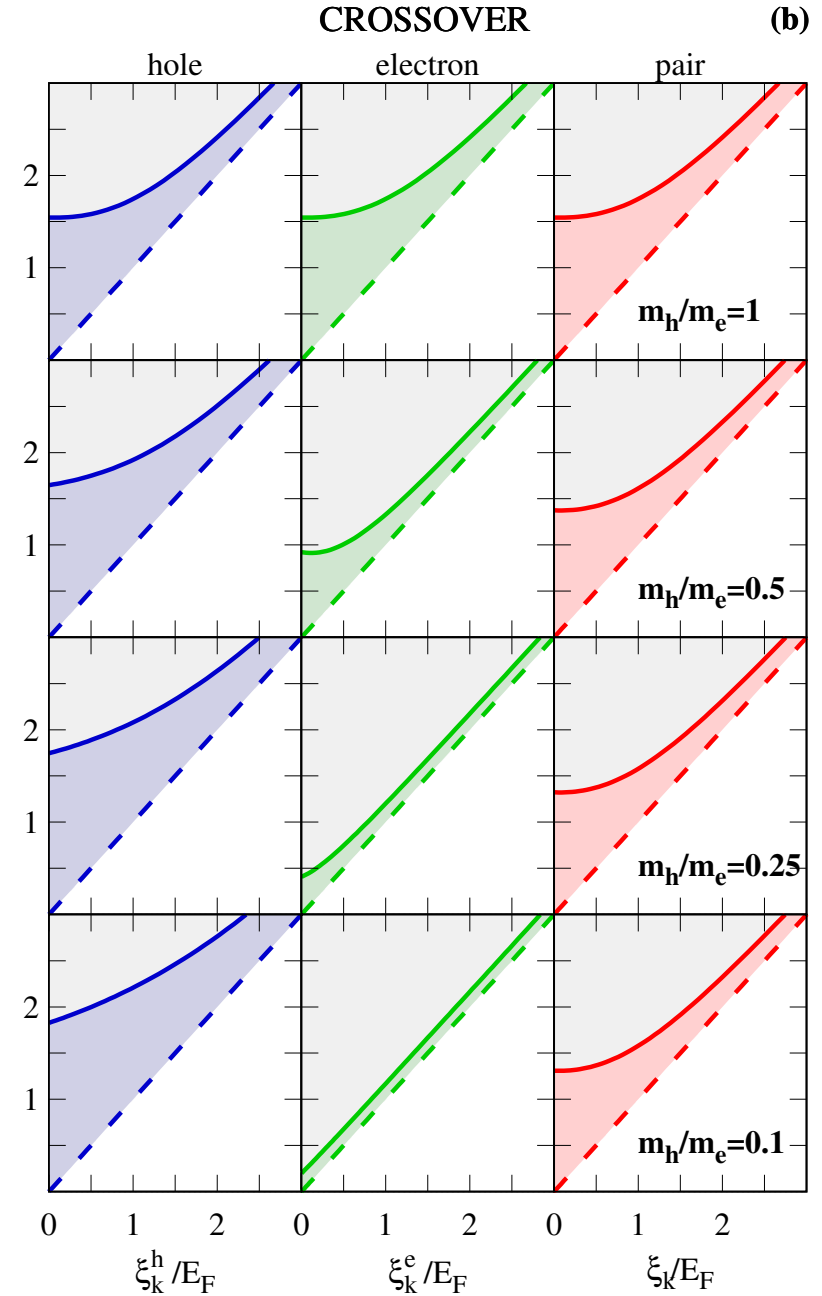

Figure 4. The dashed lines are the normal state single-particle excitation spectrum $\xi_{k}^{h}$ for the holes (blue panels), $\xi_{k}^{e}$ for the electrons (green panels) and $\xi_{k}$ for the pairs (red panels), and the solid lines the corresponding superfluid state excitation spectrum $E_{k}^{+}, E_{k}^{-}$and $E_{k}$. (a) For densities in the BEC regime, with $C F=0.95$; (b) For densities in the crossover regime close to the onset density, with $C F=0.5$.

A consequence of these results can be seen when $\Delta_{\max }$ as a function of $n$ is scaled to the electron and hole Fermi energies (Figure 5).

For equal masses, the onset density $n_{0}$ is reached when $\Delta_{\max } \sim 1.5 E_{F}$. However for unequal masses at $n_{0}, \Delta_{\max } \sim E_{F}^{h}$ but $\Delta_{\max } \gg E_{F}^{e}$, a result that at first sight is surprising. The reason is that to weaken the screening sufficiently for superfluidity to appear, $\Delta_{\max }$ must be larger than both $E_{F}^{e}$ and $E_{F}^{h}$, and only when $\Delta_{\max }>E_{F}^{h}$ will both inequalities be satisfied. The critical value for $\Delta / E_{F}^{e}$ thus depends on $E_{F}^{h} / E_{F}^{e}$, and hence on $m_{h} / m_{e}$. The smaller $m_{h} / m_{e}$, the larger the critical $\Delta / E_{F}^{e}$. 

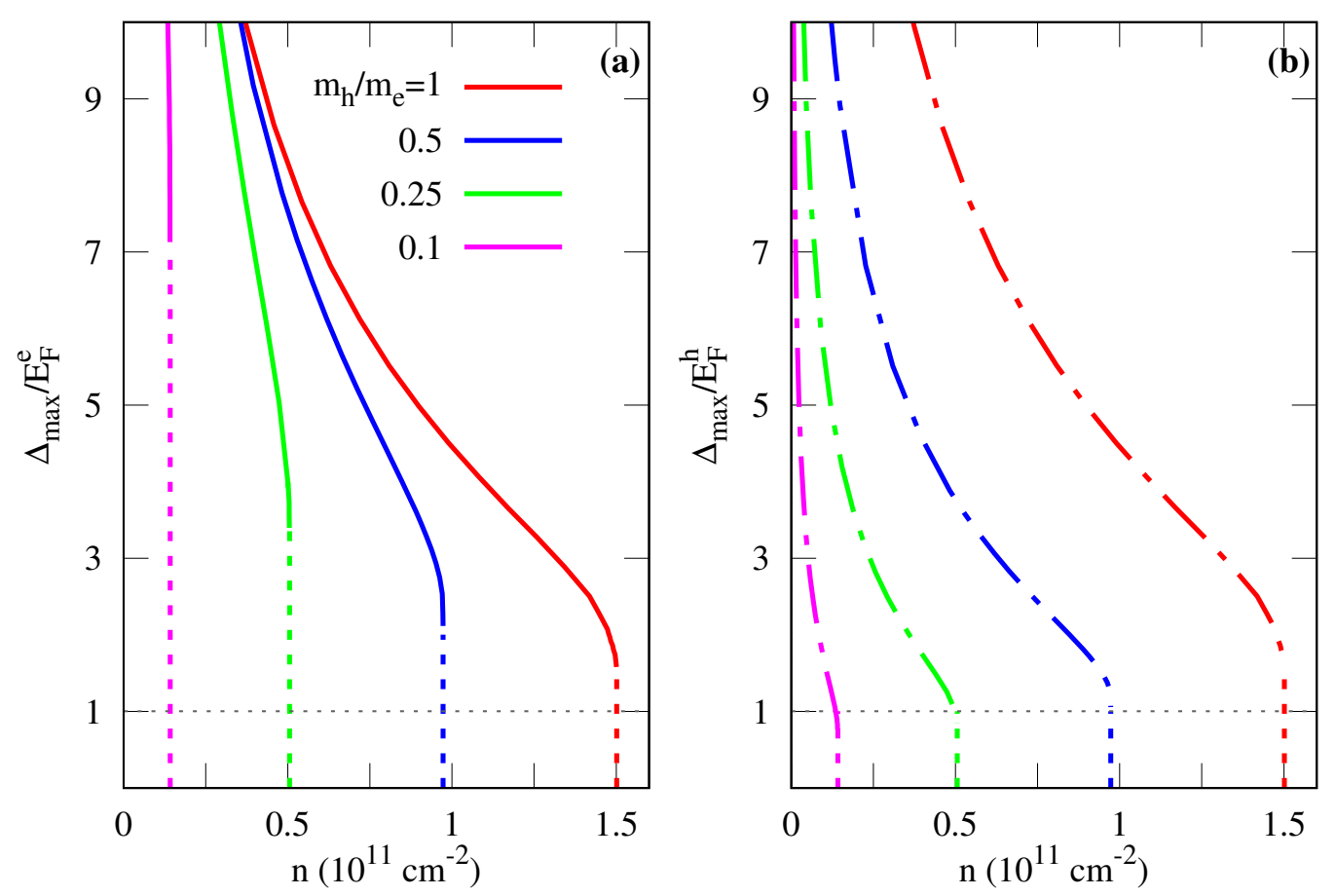

Figure 5. (a) Maximum superfluid gap $\Delta_{\max }$ scaled by the electron Fermi energies $E_{F, e}$ as a function of density. (b) $\Delta_{\max }$ scaled by the hole Fermi energies $E_{F, h}$.

\section{Discussion}

We find that the sensitivity of the electron-hole superfluid properties to unequal masses is markedly different in the BEC regime and the BEC-BCS crossover regime. In the $B E C$ regime, the system properties are sensitive only to the reduced mass of the electronhole pairs $m_{r}$, so the properties are not affected by changes in the mass ratio $m_{h} / m_{e}$, provided $m_{r}$ remains fixed. This is consistent with the picture in the BEC regime of tightly bound electron-hole pairs.

In contrast, in the BEC-BCS crossover regime, unequal masses lead to new effects. In this regime the electron-hole pairs are significantly less strongly bound when the masses are unequal. With decreasing $m_{h} / m_{e}$, the system transits from the BEC regime into the BEC-BCS crossover regime at a lower density, and the superfluidity becomes weaker. The onset density $n_{0}$ at which superfluidity is killed by screening is smaller and the maximum transition temperatures $T_{B K T}$ is lower.

The onset density occurs before the superfluidity can enter the weak-coupled BCS regime. For this reason, effects of unequal masses from mismatched Fermi seas at equal densities are not important. Away from the BCS regime, the influence of unequal masses on superfluid properties acts primarily through the screening polarizabilities.

Since in the BEC-BCS crossover regime, unlike in the BEC regime, there is a significant sensitivity to unequal masses, it is in the crossover regime that the experimental search for exotic electron-hole superfluid and supersolid phases should be conducted.

Author Contributions: Conceptualization, S.C., A.P. and D.N.; methodology, S.C. and A.P.; software, S.C.; validation, S.C. and D.N.; formal analysis, S.C. and A.P.; investigation, S.C., A.P., F.M.P. and D.N.; resources, S.C. and F.M.P.; data curation, S.C.; writing-original draft preparation, S.C. and D.N.; writing-review and editing, S.C., A.P., F.M.P. and D.N.; visualization, S.C.; supervision, A.P. and D.N.; project administration, D.N.; funding acquisition, S.C. and F.M.P. All authors have read and agreed to the published version of the manuscript.

Funding: S.C. is supported by the FWO-Vl (Fonds Wetenschappelijk Onderzoek-Vlaanderen).

Institutional Review Board Statement: Not applicable. 
Informed Consent Statement: Not applicable.

Data Availability Statement: All the data in this study are available within this paper.

Conflicts of Interest: The authors declare no conflict of interest. The funders had no role in the design of the study; in the collection, analyses, or interpretation of data; in the writing of the manuscript, or in the decision to publish the results.

\section{Abbreviations}

The following abbreviations are used in this manuscript:

BEC Bose Einstein Condensation

BCS Bardeen Cooper Schrieffer

\section{Appendix A. Polarizabilities in the Deep BEC Limit}

The deep BEC limit is characterised by a very strong superfluid gap $\Delta_{\mathbf{k}} \gg E_{F}$, with $\Delta_{\mathbf{k}}$ having its maximum at $k=0$, and remaining approximately constant out to $k \lesssim 4 k_{F}$. Thus we can assume:

(i) $\quad E_{\mathbf{k}} \sim \Delta_{\mathbf{k}}$ (Equation (3));

(ii) $\Delta_{\mathbf{k}} \gg \delta \xi_{\mathbf{k}}$, so that $E_{\mathbf{k}}^{+} \sim E_{\mathbf{k}}^{-} \sim \Delta_{\mathbf{k}}$ (Equation (8));

(iii) $\Delta_{\mathbf{k}} \simeq \Delta_{\mathbf{k}-\mathbf{q}} \simeq$ constant, for $q<2 k_{F}$.

Equations (5)-(7) for $q<2 k_{F}$ can thus be approximated:

$$
\begin{aligned}
& \Pi_{e}(\mathbf{q})=-\frac{g_{s} g_{v}}{L^{2}} \sum_{\mathbf{k}}\left[\frac{u_{\mathbf{k}}^{2} v_{\mathbf{k}-\mathbf{q}}^{2}}{\Delta_{\mathbf{k}-\mathbf{q}}+\Delta_{\mathbf{k}}}+\frac{v_{\mathbf{k}}^{2} u_{\mathbf{k}-\mathbf{q}}^{2}}{\Delta_{\mathbf{k}-\mathbf{q}}+\Delta_{\mathbf{k}}}\right]=-\frac{g_{s} g_{v}}{L^{2}} \sum_{\mathbf{k}}\left[\frac{u_{\mathbf{k}}^{2} v_{\mathbf{k}-\mathbf{q}}^{2}}{2 \Delta_{\mathbf{k}}}+\frac{v_{\mathbf{k}}^{2} u_{\mathbf{k}-\mathbf{q}}^{2}}{2 \Delta_{\mathbf{k}}}\right], \\
& \Pi_{h}(\mathbf{q})=-\frac{g_{s} g_{v}}{L^{2}} \sum_{\mathbf{k}}\left[\frac{u_{\mathbf{k}}^{2} v_{\mathbf{k}-\mathbf{q}}^{2}}{\Delta_{\mathbf{k}-\mathbf{q}}+\Delta_{\mathbf{k}}}+\frac{v_{\mathbf{k}}^{2} u_{\mathbf{k}-\mathbf{q}}^{2}}{\Delta_{\mathbf{k}-\mathbf{q}}+\Delta_{\mathbf{k}}}\right]=-\frac{g_{s} g_{v}}{L^{2}} \sum_{\mathbf{k}}\left[\frac{u_{\mathbf{k}}^{2} v_{\mathbf{k}-\mathbf{q}}^{2}}{2 \Delta_{\mathbf{k}}}+\frac{v_{\mathbf{k}}^{2} u_{\mathbf{k}-\mathbf{q}}^{2}}{2 \Delta_{\mathbf{k}}}\right], \\
& \Pi_{a}(\mathbf{q})=\frac{g_{s} g_{v}}{L^{2}} \sum_{\mathbf{k}} \frac{\Delta_{\mathbf{k}}}{2 \Delta_{\mathbf{k}}} \frac{\Delta_{\mathbf{k}-\mathbf{q}}}{2 \Delta_{\mathbf{k}-\mathbf{q}}}\left[\frac{1}{\Delta_{\mathbf{k}-\mathbf{q}}+\Delta_{\mathbf{k}}}+\frac{1}{\Delta_{\mathbf{k}-\mathbf{q}}+\Delta_{\mathbf{k}}}\right]=\frac{g_{s} g_{v}}{L^{2}} \sum_{\mathbf{k}} \frac{1}{4}\left[\frac{1}{2 \Delta_{\mathbf{k}}}+\frac{1}{2 \Delta_{\mathbf{k}}}\right] .
\end{aligned}
$$

The Bogoliubov amplitude products can be approximated,

$$
u_{\mathbf{k}}^{2} v_{\mathbf{k}-\mathbf{q}}^{2}+v_{\mathbf{k}}^{2} u_{\mathbf{k}-\mathbf{q}}^{2}=\frac{1}{2}\left(1+\frac{\xi_{\mathbf{k}}}{E_{\mathbf{k}}} \frac{\xi_{\mathbf{k}-\mathbf{q}}}{E_{\mathbf{k}-\mathbf{q}}}\right) \sim \frac{1}{2}\left(1+\frac{\xi_{\mathbf{k}}^{2}}{\Delta_{\mathbf{k}}^{2}}\right) \sim \frac{1}{2},
$$

so that Equations (A1)-(A3) become:

$$
\Pi_{e}(\mathbf{q})=\Pi_{h}(\mathbf{q})=-\Pi_{a}(\mathbf{q})=-\frac{g_{s} g_{v}}{L^{2}} \sum_{\mathbf{k}} \frac{1}{4 \Delta_{\mathbf{k}}} .
$$

Equation (A5) shows in the BEC limit that the normal and anomalous polarizabilities have the same $q$-dependence. They are driven purely by the superfluid gap $\Delta_{\mathbf{k}}$ and so are approximately constant out to $q<2 k_{F}$, as shown in Figure 3a. Importantly, because $\Delta_{\mathbf{k}}$ depends only on the reduced mass $m_{r}$, the polarizabilities are insensitive to the mass ratio $m_{h} / m_{e}$ in the BEC regime.

\section{Appendix B. Polarizabilities in the BCS Limit}

The BCS limit is characterised by a weak superfluid gap $\Delta_{\mathbf{k}}<E_{F}$ with a narrow peak at $k=k_{F}$. To calculate $\Pi_{e}(\mathbf{q})$ and $\Pi_{h}(\mathbf{q})$ in this limit, we expand the excitation energies (Equations (3) and (8)): 


$$
E_{\mathbf{k}}=\sqrt{\xi_{\mathbf{k}}^{2}+\Delta_{\mathbf{k}}^{2}}=\left|\xi_{\mathbf{k}}\right|\left(1+\frac{\Delta_{\mathbf{k}}^{2}}{2 \xi_{\mathbf{k}}^{2}}\right), \quad E_{\mathbf{k}}^{ \pm}=\left|\xi_{\mathbf{k}}\right|\left(1+\frac{\Delta_{\mathbf{k}}^{2}}{2 \xi_{\mathbf{k}}^{2}}\right) \pm \delta \xi_{\mathbf{k}} .
$$

In the BCS limit, the Bogoliubov amplitudes are approximately step functions, $u_{\mathbf{k}}^{2} \sim \Theta\left(k-k_{F}\right)$ and $v_{\mathbf{k}}^{2} \sim \Theta\left(k_{F}-k\right)$ and the product $u_{\mathbf{k}}^{2} v_{\mathbf{k}-\mathbf{q}}^{2}\left(v_{\mathbf{k}}^{2} u_{\mathbf{k}-\mathbf{q}}^{2}\right)$ is only non-zero for $k>k_{F}$ and $k-q<k_{F}\left(k<k_{F}\right.$ and $\left.k-q>k_{F}\right)$. We calculate the $\Pi_{\mathcal{e}}(\mathbf{q})$ and $\Pi_{h}(\mathbf{q})$ for these two cases.

For $k>k_{F}$ and $k-q<k_{F}, u_{\mathbf{k}}^{2} v_{\mathbf{k}-\mathbf{q}}^{2}=1$ and $v_{\mathbf{k}}^{2} u_{\mathbf{k}-\mathbf{q}}^{2}=0$, so Equations (5) and (6) become

$$
\begin{aligned}
& \Pi_{e}(\mathbf{q})=-\frac{g_{s} g_{v}}{L^{2}} \sum_{\mathbf{k}}\left[\frac{1}{E_{\mathbf{k}-\mathbf{q}}^{+}+E_{\mathbf{k}}^{-}}\right], \\
& \Pi_{h}(\mathbf{q})=-\frac{g_{s} g_{v}}{L^{2}} \sum_{\mathbf{k}}\left[\frac{1}{E_{\mathbf{k}-\mathbf{q}}^{-}+E_{\mathbf{k}}^{+}}\right] .
\end{aligned}
$$

Using Equation (A6), for $k>k_{F},\left|\xi_{\mathbf{k}}\right|=\xi_{\mathbf{k}}$ :

$$
\begin{aligned}
& E_{\mathbf{k}}^{+}=\xi_{\mathbf{k}}+\delta \xi_{\mathbf{k}}+\frac{\Delta_{\mathbf{k}}^{2}}{2 \xi_{\mathbf{k}}}=\xi_{\mathbf{k}}^{h}+\frac{\Delta_{\mathbf{k}}^{2}}{2 \xi_{\mathbf{k}}}, \\
& E_{\mathbf{k}}^{-}=\xi_{\mathbf{k}}-\delta \xi_{\mathbf{k}}+\frac{\Delta_{\mathbf{k}}^{2}}{2 \xi_{\mathbf{k}}}=\xi_{\mathbf{k}}^{e}+\frac{\Delta_{\mathbf{k}}^{2}}{2 \xi_{\mathbf{k}}},
\end{aligned}
$$

and for $k-q<k_{F},\left|\xi_{\mathbf{k}-\mathbf{q}}\right|=-\xi_{\mathbf{k}-\mathbf{q}}$ :

$$
\begin{aligned}
& E_{\mathbf{k}-\mathbf{q}}^{+}=-\xi_{\mathbf{k}-\mathbf{q}}+\delta \xi_{\mathbf{k}-\mathbf{q}}-\frac{\Delta_{\mathbf{k}-\mathbf{q}}^{2}}{2 \xi_{\mathbf{k}-\mathbf{q}}}=-\xi_{\mathbf{k}-\mathbf{q}}^{e}-\frac{\Delta_{\mathbf{k}-\mathbf{q}}^{2}}{2 \xi_{\mathbf{k}-\mathbf{q}}}, \\
& E_{\mathbf{k}-\mathbf{q}}^{-}=-\xi_{\mathbf{k}-\mathbf{q}}-\delta \xi_{\mathbf{k}-\mathbf{q}}-\frac{\Delta_{\mathbf{k}-\mathbf{q}}^{2}}{2 \xi_{\mathbf{k}-\mathbf{q}}}=-\xi_{\mathbf{k}-\mathbf{q}}^{h}-\frac{\Delta_{\mathbf{k}-\mathbf{q}}^{2}}{2 \xi_{\mathbf{k}-\mathbf{q}}} .
\end{aligned}
$$

Equations (A7) and (A8) become:

$$
\begin{aligned}
& \Pi_{e}(\mathbf{q})=-\frac{2}{L^{2}} \sum_{\mathbf{k}}\left[\frac{1}{\xi_{\mathbf{k}}^{e}-\xi_{\mathbf{k}-\mathbf{q}}^{e}+\frac{\Delta_{\mathbf{k}}^{2}}{2 \xi_{\mathbf{k}}}-\frac{\Delta_{\mathbf{k}-\mathbf{q}}^{2}}{2 \xi_{\mathbf{k}-\mathbf{q}}}}\right], \\
& \Pi_{h}(\mathbf{q})=-\frac{2}{L^{2}} \sum_{\mathbf{k}}\left[\frac{1}{\xi_{\mathbf{k}}^{h}-\xi_{\mathbf{k}-\mathbf{q}}^{h}+\frac{\Delta_{\mathbf{k}}^{2}}{2 \xi_{\mathbf{k}}}-\frac{\Delta_{\mathbf{k}-\mathbf{q}}^{2}}{2 \xi_{\mathbf{k}-\mathbf{q}}}}\right] .
\end{aligned}
$$

In the second case, for $k<k_{F}$ and $k-q>k_{F}, u_{\mathbf{k}}^{2} v_{\mathbf{k}-\mathbf{q}}^{2}=0$ and $v_{\mathbf{k}}^{2} u_{\mathbf{k}-\mathbf{q}}^{2}=1$, so:

$$
\begin{aligned}
& \Pi_{e}(\mathbf{q})=-\frac{2}{L^{2}} \sum_{\mathbf{k}}\left[\frac{1}{\xi_{\mathbf{k}-\mathbf{q}}^{e}-\xi_{\mathbf{k}}^{e}+\frac{\Delta_{\mathbf{k}-\mathbf{q}}^{2}}{2 \xi_{\mathbf{k}-\mathbf{q}}}-\frac{\Delta_{\mathbf{k}}^{2}}{2 \xi_{\mathbf{k}}}}\right], \\
& \Pi_{h}(\mathbf{q})=-\frac{2}{L^{2}} \sum_{\mathbf{k}}\left[\frac{1}{\xi_{\mathbf{k}-\mathbf{q}}^{h}-\xi_{\mathbf{k}}^{h}+\frac{\Delta_{\mathbf{k}-\mathbf{q}}^{2}}{2 \xi_{\mathbf{k}-\mathbf{q}}}-\frac{\Delta_{\mathbf{k}}^{2}}{2 \xi_{\mathbf{k}}}}\right] .
\end{aligned}
$$

Combining Equations (A13) and (A15) for $\Pi_{e}(\mathbf{q})$ and Equations (A14) and (A16) for $\Pi_{h}(\mathbf{q})$, Equations (5) and (6) in this limit become: 


$$
\begin{aligned}
& \Pi_{e}(\mathbf{q})=-\frac{2}{L^{2}} \sum_{\mathbf{k}}\left[\frac{\Theta\left(k-k_{F}\right)-\Theta\left(k-q-k_{F}\right)}{\xi_{\mathbf{k}}^{e}-\xi_{\mathbf{k}-\mathbf{q}}^{e}+\frac{\Delta_{\mathbf{k}}^{2}}{2 \xi_{\mathbf{k}}}-\frac{\Delta_{\mathbf{k}-\mathbf{q}}^{2}}{2 \xi_{\mathbf{k}-\mathbf{q}}}}\right], \\
& \Pi_{h}(\mathbf{q})=-\frac{2}{L^{2}} \sum_{\mathbf{k}}\left[\frac{\Theta\left(k-k_{F}\right)-\Theta\left(k-q-k_{F}\right)}{\xi_{\mathbf{k}}^{h}-\xi_{\mathbf{k}-\mathbf{q}}^{h}+\frac{\Delta_{\mathbf{k}}^{2}}{2 \xi_{\mathbf{k}}}-\frac{\Delta_{\mathbf{k}-\mathbf{q}}^{2}}{2 \xi_{\mathbf{k}-\mathbf{q}}}}\right] .
\end{aligned}
$$

Thus, in this limit, the $\Pi_{e}(\mathbf{q})$ depends only on electron energy terms and $\Pi_{h}(\mathbf{q})$ depends only on hole energy terms. This is the reason why with mass imbalance, $\Pi_{e}(\mathbf{q})$ and $\Pi_{h}(\mathbf{q})$ behave significantly differently (Figure $3 \mathbf{b}$ ).

Furthermore, by applying a binomial expansion,

$$
\begin{aligned}
& \Pi_{e}(\mathbf{q})=-\frac{g_{s} g_{v}}{L^{2}} \sum_{\mathbf{k}}\left[\frac{\Theta\left(k-k_{F}\right)-\Theta\left(k-q-k_{F}\right)}{\tilde{\zeta}_{\mathbf{k}}^{e}-\tilde{\xi}_{\mathbf{k}-\mathbf{q}}^{e}} \frac{1}{1+\frac{1}{\xi_{\mathbf{k}}^{e}-\tilde{\xi}_{\mathbf{k}-\mathbf{q}}^{e}}\left(\frac{\Delta_{\mathbf{k}}^{2}}{2 \xi_{\mathbf{k}}}-\frac{\Delta_{\mathbf{k}-\mathbf{q}}^{2}}{2 \xi_{\mathbf{k}-\mathbf{q}}}\right)}\right] \\
& =-\frac{g_{s} g_{v}}{L^{2}} \sum_{\mathbf{k}}\left[\frac{\Theta\left(k-k_{F}\right)-\Theta\left(k-q-k_{F}\right)}{\xi_{\mathbf{k}}^{e}-\xi_{\mathbf{k}-\mathbf{q}}^{e}}\left(1-\frac{1}{\xi_{\mathbf{k}}^{e}-\xi_{\mathbf{k}-\mathbf{q}}^{e}}\left(\frac{\Delta_{\mathbf{k}}^{2}}{2 \xi_{\mathbf{k}}}-\frac{\Delta_{\mathbf{k}-\mathbf{q}}^{2}}{2 \xi_{\mathbf{k}-\mathbf{q}}}\right)\right)\right] \\
& \Pi_{h}(\mathbf{q})=-\frac{g_{s} g_{v}}{L^{2}} \sum_{\mathbf{k}}\left[\frac{\Theta\left(k-k_{F}\right)-\Theta\left(k-q-k_{F}\right)}{\xi_{\mathbf{k}}^{h}-\xi_{\mathbf{k}-\mathbf{q}}^{h}}\left(1-\frac{1}{\xi_{\mathbf{k}}^{h}-\xi_{\mathbf{k}-\mathbf{q}}^{h}}\left(\frac{\Delta_{\mathbf{k}}^{2}}{2 \xi_{\mathbf{k}}}-\frac{\Delta_{\mathbf{k}-\mathbf{q}}^{2}}{2 \xi_{\mathbf{k}-\mathbf{q}}}\right)\right)\right] .
\end{aligned}
$$

We see in the small $\Delta_{\mathbf{k}}$ limit, that $\Pi_{e}(\mathbf{q})$ and $\Pi_{h}(\mathbf{q})$ are the Lindhard functions for the normal state [21] minus a correction term that is quadratically proportional to $\Delta_{\mathbf{k}}$.

For the anomalous polarizability, using Equations (7) and (8) can be written as,

$$
\begin{array}{r}
\Pi_{a}(\mathbf{q})=\frac{g_{s} g_{v}}{L^{2}} \sum_{\mathbf{k}} \frac{\Delta_{\mathbf{k}}}{2 E_{\mathbf{k}} \frac{\Delta_{\mathbf{k}-\mathbf{q}}}{2 E_{\mathbf{k}-\mathbf{q}}}}\left[\frac{1}{E_{\mathbf{k}-\mathbf{q}}+E_{\mathbf{k}}-\frac{1}{2}\left(\xi_{\mathbf{k}-\mathbf{q}}^{h}-\xi_{\mathbf{k}-\mathbf{q}}^{e}\right)+\frac{1}{2}\left(\xi_{\mathbf{k}}^{h}-\xi_{\mathbf{k}}^{e}\right)}\right. \\
\left.+\frac{1}{E_{\mathbf{k}-\mathbf{q}}+E_{\mathbf{k}}+\frac{1}{2}\left(\xi_{\mathbf{k}-\mathbf{q}}^{h}-\xi_{\mathbf{k}-\mathbf{q}}^{e}\right)-\frac{1}{2}\left(\xi_{\mathbf{k}}^{h}-\xi_{\mathbf{k}}^{e}\right)}\right] .
\end{array}
$$

For large mass imbalance $m_{e} \gg m_{h}$, we can neglect $\xi_{\mathbf{k}}^{e}$ in Equation (A21) compared with $\xi_{\mathbf{k}}^{h}$. Recalling that $E_{\mathbf{k}}$ depends only on $m_{r}$ and $\xi_{\mathbf{k}}^{h}$ depends only on $m_{h}$, and that $m_{r} \simeq m_{h}$, we conclude that $\Pi_{a}(\mathbf{q})$ is driven by the lighter mass $m_{h}$, and hence that it tracks $\Pi_{h}(\mathbf{q})$.

\section{References}

1. Iskin, M. Vortex core states in superfluid Fermi-Fermi mixtures with unequal masses. Phys. Rev. A 2008, 78, 021604. [CrossRef]

2. Wang, J.; Che, Y.; Zhang, L.; Chen, Q. Enhancement effect of mass imbalance on Fulde-Ferrell-Larkin-Ovchinnikov type of pairing in Fermi-Fermi mixtures of ultracold quantum gases. Sci. Rep. 2017, 7, 39783. [CrossRef] [PubMed]

3. Forbes, M.M.; Gubankova, E.; Liu, W.V.; Wilczek, F. Stability criteria for breached-pair superfluidity. Phys. Rev. Lett. 2005, 94, 017001. [CrossRef]

4. Zhang, Y.C.; Maucher, F.; Pohl, T. Supersolidity around a critical point in dipolar Bose-Einstein Condensates. Phys. Rev. Lett. 2019, 123, 015301. [CrossRef]

5. Kinnunen, J.J.; Baarsma, J.E.; Martikainen, J.P.; Törmä, P. The Fulde-Ferrell-Larkin-Ovchinnikov state for ultracold fermions in lattice and harmonic potentials: A review. Rep. Prog. Phys. 2018, 81, 046401. [CrossRef] [PubMed] 
6. Frank, B.; Lang, J.; Zwerger, W. Universal phase diagram and scaling functions of imbalanced Fermi gases. J. Exp. Theor. Phys. 2018, 127, 812-825. [CrossRef]

7. Pieri, P.; Neilson, D.; Strinati, G.C. Effects of density imbalance on the BCS-BEC crossover in semiconductor electron-hole bilayers. Phys. Rev. B 2007, 75, 113301. [CrossRef]

8. Subasi, A.L.; Pieri, P.; Senatore, G.; Tanatar, B. Stability of Sarma phases in density imbalanced electron-hole bilayer systems. Phys. Rev. B 2010, 81, 075436. [CrossRef]

9. Parish, M.M.; Marchetti, F.M.; Littlewood, P.B. Supersolidity in electron-hole bilayers with a large density imbalance. EPL Europhys. Lett. 2011, 95, 27007. [CrossRef]

10. Perali, A.; Neilson, D.; Hamilton, A.R. High-temperature superfluidity in double-bilayer graphene. Phys. Rev. Lett. 2013, 110, 146803. [CrossRef] [PubMed]

11. Saberi-Pouya, S.; Conti, S.; Perali, A.; Croxall, A.F.; Hamilton, A.R.; Peeters, F.M.; Neilson, D. Experimental conditions for the observation of electron-hole superfluidity in GaAs heterostructures. Phys. Rev. B 2020, 101, 140501. [CrossRef]

12. Conti, S.; Pouya, S.S.; Perali, A.; Virgilio, M.; Peeters, F.M.; Hamilton, A.R.; Scappucci, G.; Neilson, D. Electron-hole superfluidity in strained Si/Ge type II heterojunctions. arXiv 2020, arXiv:2012.05631.

13. Conti, S.; Neilson, D.; Peeters, F.M.; Perali, A. Transition metal dichalcogenides as strategy for high temperature electron-hole superfluidity. Condens. Matter 2020, 5, 22. [CrossRef]

14. Du, L.; Li, X.; Lou, W.; Sullivan, G.; Chang, K.; Kono, J.; Du, R.R. Evidence for a topological excitonic insulator in InAs/GaSb bilayers. Nat. Commun. 2017, 8, 1971. [CrossRef] [PubMed]

15. Lozovik, Y.E.; Ogarkov, S.L.; Sokolik, A.A. Condensation of electron-hole pairs in a two-layer graphene system: Correlation effects. Phys. Rev. B 2012, 86, 045429. [CrossRef]

16. Neilson, D.; Perali, A.; Hamilton, A.R. Excitonic superfluidity and screening in electron-hole bilayer systems. Phys. Rev. B 2014, 89, 060502. [CrossRef]

17. Salasnich, L.; Manini, N.; Parola, A. Condensate fraction of a Fermi gas in the BCS-BEC crossover. Phys. Rev. A 2005, 72, 023621. [CrossRef]

18. Kosterlitz, J.M.; Thouless, D.J. Ordering, metastability and phase transitions in two-dimensional systems. J. Phys. C Solid State Phys. 1973, 6, 1181-1203. [CrossRef]

19. Conti, S.; Perali, A.; Peeters, F.M.; Neilson, D. Multicomponent electron-hole superfluidity and the BCS-BEC crossover in double bilayer graphene. Phys. Rev. Lett. 2017, 119, 257002. [CrossRef] [PubMed]

20. López Ríos, P.; Perali, A.; Needs, R.J.; Neilson, D. Evidence from quantum Monte Carlo simulations of large-gap superfluidity and BCS-BEC crossover in double electron-hole layers. Phys. Rev. Lett. 2018, 120, 177701. [CrossRef] [PubMed]

21. Giuliani, G.; Vignale, G. Quantum Theory of the Electron Liquid; Masters Series in Physics and Astronomy; Cambridge University Press: Cambridge, UK, 2005. 